\title{
GnRH antagonist for patients with polycystic ovary syndrome undergoing controlled ovarian hyperstimulation for in vitro fertilization and embryo transfer in fresh cycles
}

\author{
XIANG-HONG ZHAI ${ }^{1,2}$, PING ZHANG ${ }^{2,3}$, FENG-XIA WU ${ }^{4}$, AN-CONG WANG ${ }^{2,3 *}$ and PEI-SHU LIU ${ }^{5 *}$ \\ ${ }^{1}$ Department of Obstetrics and Gynecology, Linyi Health School; Departments of ${ }^{2}$ Reproductive Medicine and \\ ${ }^{3}$ Obstetrics and Gynecology, Linyi People's Hospital, Linyi, Shandong 276003; ${ }^{4}$ Department of Anatomy, Shandong University; \\ ${ }^{5}$ Department of Obstetrics and Gynecology, Qilu Hospital, Shandong University, Jinan, Shandong 250012, P.R. China
}

Received September 27, 2015; Accepted February 1, 2017

DOI: $10.3892 /$ etm.2017.4309

\begin{abstract}
The aim of the present study was to evaluate the influence of a gonadotropin-releasing hormone $(\mathrm{GnRH})$ antagonist compared with a GnRH agonist on the in vitro fertilization cycle outcome in patients with polycystic ovary syndrome. The outcomes of pregnancy were evaluated. The area under the curve (AUC) of the receiver operating characteristic (ROC) curve was also used to evaluate whether the endometrial thickness $(\mathrm{cm})$ and estradiol $\left(\mathrm{E}_{2}\right)$ level $(\mathrm{pg} / \mathrm{ml})$ on the day of human chorionic gonadotropin (hCG) administration (the hCG day) had the best sensitivity and specificity for predicting a clinical pregnancy. The results demonstrated that there were significant differences in the $\mathrm{E}_{2}$ and progesterone levels between the two treatment groups on the hCG day. Furthermore, the mean number of total oocytes retrieved, mean number of 2 pronuclei oocytes, mean number of oocytes cleaved $(\mathrm{P}<0.05)$, mean number of embryos available $(\mathrm{P}=0.022)$ and mean number of embryos transferred $(\mathrm{P}=0.014)$ were significantly different. Additionally, the rates of ectopic pregnancy $(\mathrm{P}=0.984)$ and ovarian hyperstimulation syndrome $(\mathrm{P}=0.976)$ did not differ significantly between the treatment groups. Although the biochemical pregnancy $(\mathrm{P}=0.592)$, clinical pregnancy $(\mathrm{P}=0.617)$ and live birth $(\mathrm{P}=0.365)$ rates were lower with the GnRH antagonist than with the GnRH agonist, there were no significant differences in the outcomes between the two groups. Analysis of the influence of endometrial thickness with respect to the clinical pregnancy using
\end{abstract}

Correspondence to: Dr An-Cong Wang, Department of Reproductive Medicine, Linyi People's Hospital, 27 Jiefang Road, Linyi, Shandong 276003, P.R. China

E-mail: ancongw12@163.com

*Contributed equally

Key words: polycystic ovary syndrome, in vitro fertilization and embryo transfer, gonadotrophin-releasing hormone antagonist, ovarian hyperstimulation syndrome, clinical pregnancy, fresh cycle the ROC (AUC) method revealed that when the best cutoff of $9.75 \mathrm{~cm}$ was used, the sensitivity was $62.5 \%$, the specificity was $43.1 \%$ and the AUC was 0.53. Additionally, the Youden index was 0.056. Analysis of the influence of the $\mathrm{E}_{2}$ level on the hCG day on clinical pregnancy, using the ROC (AUC) method showed that the best cutoff was $2,984.5 \mathrm{pg} / \mathrm{ml}$, which had a sensitivity of $68.8 \%$ and specificity of $52.9 \%$, while the AUC was 0.573 (with a Youden index of 0.217). Furthermore, the results demonstrated that neither the endometrial thickness nor the $\mathrm{E}_{2}$ level on the hCG day had the best sensitivity and specificity for predicting a clinical pregnancy.

\section{Introduction}

Polycystic ovary syndrome (PCOS) is the most frequent endocrinopathy in women of reproductive age. Hyperandrogenism, polycystic ovaries and chronic anovulation without other specific underlying diseases of the adrenal or pituitary glands are the main features of the disease (1). PCOS is associated with long-term sequelae, including latent diabetes, hypertension, cardiovascular disease and even endometrial carcinoma. Different combinations of clinical and biological criteria as well as ultrasound have been proposed for diagnosis. Since the etiology of the disease remains poorly understood and the effective treatment for subfertile patients with PCOS has not been elucidated, numerous controlled ovarian hyperstimulation $(\mathrm{COH})$ strategies have become available for the treatment of patients with PCOS who were undergoing in vitro fertilization and embryo transfer (IVF-ET) with the use of gonadotropins (2).

The current $\mathrm{COH}$ strategies for IVF-ET pursue three main objectives, namely, hypophyseal activity suppression, multiple follicle growth stimulation and ovulation induction. In comparison with natural cycles, $\mathrm{COH}$ results in high pregnancy and implantation rates $(3,4)$. However, gonadotropin use is frequently associated with premature luteinizing hormone (LH) surging prior to oocyte retrieval. To prevent an untimely LH surge and enable appropriate development of the leading follicles, hypophyseal activity may be suppressed using two types of drugs: Gonadotrophin-releasing hormone $(\mathrm{GnRH})$ agonists and GnRH antagonists. The mechanisms of action are 
entirely different, although the two drugs share similar clinical indications (5). It is almost 20 years since GnRH antagonists were first applied in clinical practice. However, the safety and effectiveness of GnRH antagonists for IVF-ET remain unclear.

Ovarian hyperstimulation syndrome (OHSS) is a serious complication of $\mathrm{COH}$, particularly in patients with PCOS who are undergoing COS. OHSS is an exaggerated response to ovulation induction with either exogenous or endogenous human chorionic gonadotropin (hCG). It has been demonstrated that in patients who are at high risk for severe OHSS, a GnRH antagonist should be selected for use during the first IVF-ET because it enables the use of a GnRH agonist, instead of hCG, to trigger ovulation, with the consequent elimination of severe OHSS (6).

Although some randomized controlled trials (RCTs) have compared GnRH antagonists with GnRH agonists in patients with PCOS, there is no consensus as to which protocol is better, the GnRH antagonist or GnRH agonist. The aim of the present study was to evaluate the effects of a $\mathrm{GnRH}$ antagonist in comparison with those of a GnRH agonist on IVF cycle outcomes in patients with PCOS. However, the main aim was to help to aid fertility specialists in the decision-making process for infertile patients with PCOS.

\section{Materials and methods}

Patients. The computerized files (clinical medicine reproductive management system, CCRM) of the patients with PCOS who were admitted to the IVF unit of Linyi People's Hospital (Linyi, China) between December 25, 2013 and April 30, 2014, all of whom had reached the ovum pick-up stage were reviewed. The study was approved by the Ethics Committee of Linyi People's Hospital. Additionally, all of patients could enter the study by meeting the following requirements: Aged $22-45$ years, with a body mass index (BMI) of $18-33 \mathrm{~kg} / \mathrm{m}^{2}$ and transplantation of fresh cycles. The fecundities of all male partners of the patients were normal according to World Health Organization criteria (7).

In the present study, patients with PCOS were analyzed based on the Rotterdam 2003 consensus criteria, for the presence of two of the following three features: Oligoand/or anovulation, clinical and/or biochemical signs of hyperandrogenism, polycystic ovaries and exclusion of other endocrinopathies such as thyroid disease and prolactin excess (1). All of the patients underwent $\mathrm{COH}$ using either the midluteal long $\mathrm{GnRH}$ agonist [triptorelin; Ferring $\mathrm{GmbH}$, Kiel, Germany; $0.1 \mathrm{mg} /$ day, subcutaneously (SC)] suppressive protocol (agonist group, group $\mathrm{GnRH}-\mathrm{a}$ ) or the multiple-dose fixed GnRH antagonist [Cetrotide (cetrorelix acetate); Merck-Serono Ltd., Aubonne, Switzerland; 0.25 mg/day SC) protocol (antagonist group, GnRH-A).

Data on the baseline characteristics of patients in the agonist and antagonist groups were collected from the files. Serum follicle-stimulating hormone (FSH) and LH levels were measured with an ELISA kit (KAQ0841; Invitrogen; Thermo Fisher Scientific, Inc., Waltham, MA, USA). $E_{2}$ level was evaluated using the Enhanced Estradiol (eE2) kit (cat. no. 127023; Siemens AG, Munich, Germany) and progesterone (P) level was evaluated using the Progesterone PRGE kit (cat. no. 059262; Siemens AG).
All patients underwent baseline transvaginal sonography on day 2 or 3 of the menstrual cycle to check the antral follicle count and the thickness of the endometrium. In the GnRH-a group, a protocol was started from day 21 of the menstrual cycle (following the oral contraceptive Diane-35 for 18 days on day 3 of the menstrual cycle) with an injection of triptorelin $0.1 \mathrm{mg} \mathrm{SC}$ once daily for 10 days, and the dosage was later reduced to $0.05 \mathrm{mg}$ daily. When downregulation was achieved, injection of recombinant FSH ( $\mathrm{rFSH}$; Gonal F, Merck-Serono Ltd.) was initiated at a dosage of 75-225 IU. The dose of rFSH was then adjusted 4 days after stimulation depending on the ovarian response, as assessed by a transvaginal scan and the serum estradiol levels. Additionally, when the diameter of the largest follicle was $\geq 16 \mathrm{~mm}, 75-150$ IU rLH- $\alpha$ (Luveris; Merck-Serono Ltd.) was injected subcutaneously once daily. The doses of $\mathrm{rFSH}$ and $\mathrm{rLH}-\alpha$ were adjusted according to the serum estradiol levels and the growth of the ovarian follicle, which was monitored by serial ultrasonography. Treatment with triptorelin, $\mathrm{rFSH}$ and $\mathrm{rLH}-\alpha$ was continued until the day of the final oocyte maturation trigger.

In the multiple-dose fixed antagonist protocol (GnRH-A group), the injection of rFSH (Gonal F; Merck-Serono Ltd.) was started on day 2 of the menstrual cycle (75-225 IU daily). The GnRH antagonist was $0.25 \mathrm{mg}$ cetrorelix acetate, following intramuscular injection on day 6 of the menstrual cycle. Treatment with rFSH and cetrorelix acetate was then continued until the day of the final oocyte maturation trigger.

For the two groups, the final oocyte maturation trigger was the injection of $250 \mu \mathrm{g}$ human chorionic gonadotropin $\alpha$ [r-HCG (Ovidrel); Merck-Serono Ltd.]. A total of 37-38 h after rHCG injection, oocyte aspiration was performed, which was guided by transvaginal ultrasound. Embryo transfer was performed on day $3 / 5$ following oocyte retrieval. All cycles received luteal phase support with progesterone in the form of vaginal suppositories at a dose of $90 \mathrm{mg}$ once daily (Crinone; Fleet Laboratories Ltd., Northwood, UK). Clinical pregnancy was defined as visualization of a gestational sac and fetal cardiac activity on transvaginal ultrasound following 4-5 weeks of IVF-ET. The GnRH-a and GnRH-A stimulation protocols are depicted in Figs. 1 and 2.

Statistical analysis. SPSS 23.0 software for Windows was used for statistical analysis (IBM SPSS, Armonk, NY, USA). Data are reported as the mean \pm standard deviation. Differences in the variables between the two groups were statistically analyzed using Student's t-test or Wilcoxon-Mann-Whitney test and Pearson's $\chi^{2} /$ Fisher Exact tests at a two-sided significance level of 0.05 . In the present study, the area under the curve (AUC) of the receiver operating characteristic (ROC) curve was used to evaluate whether the endometrial thickness $(\mathrm{cm})$ and $\mathrm{E}_{2}$ level $(\mathrm{pg} / \mathrm{ml})$ on the day of hCG administration had the best sensitivity and specificity for predicting clinical pregnancy.

\section{Results}

Baseline characteristics and clinical outcomes of patients in the GnRH-A and GnRH-a groups for IVF. A total of 99 IVF fresh cycles with PCOS were evaluated. These comprised 50 fresh cycles in the antagonist group (GnRH-A) and 


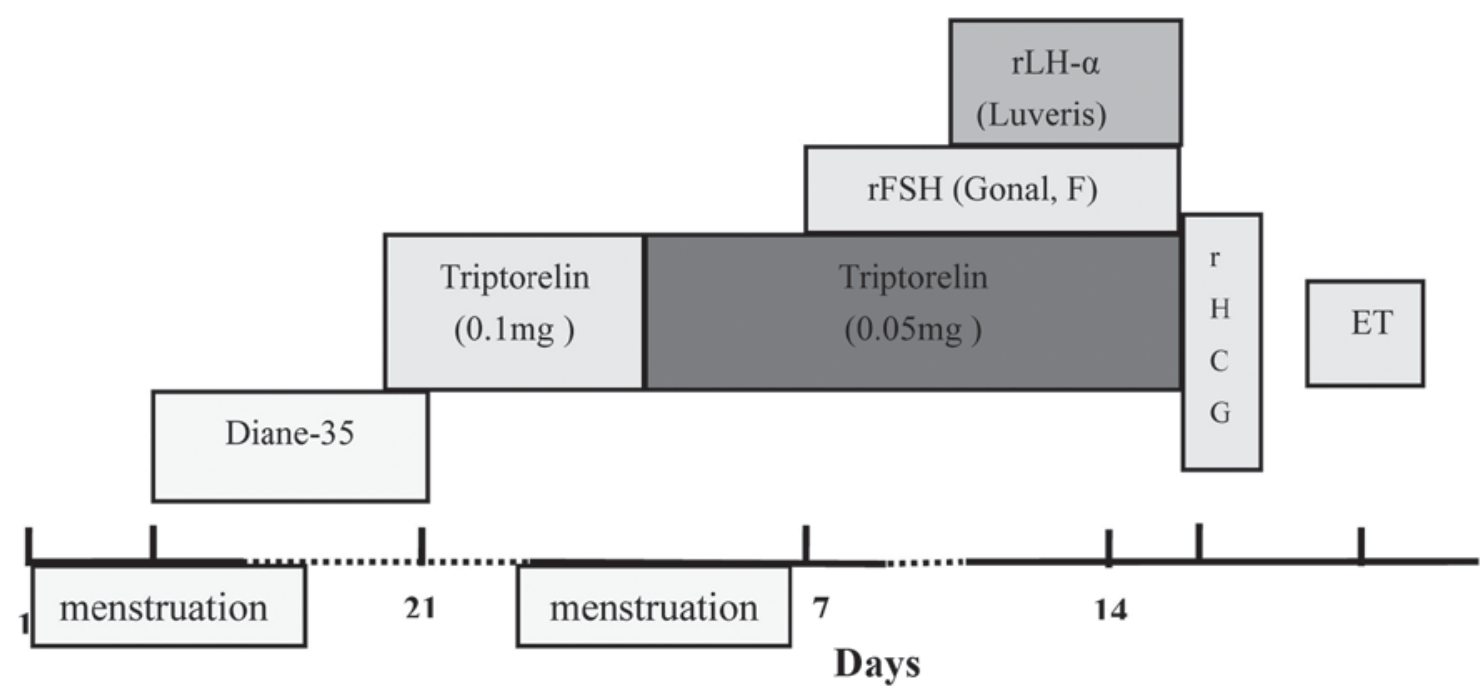

Figure 1. Gonadotropin-releasing hormone agonist stimulation protocol. rLH- $\alpha$, recombinant luteinizing hormone $\alpha$; rFSH, recombinant follicle-stimulating hormone; rHCG, recombinant human chorionic gonadotropin; ET, embryo transfer.

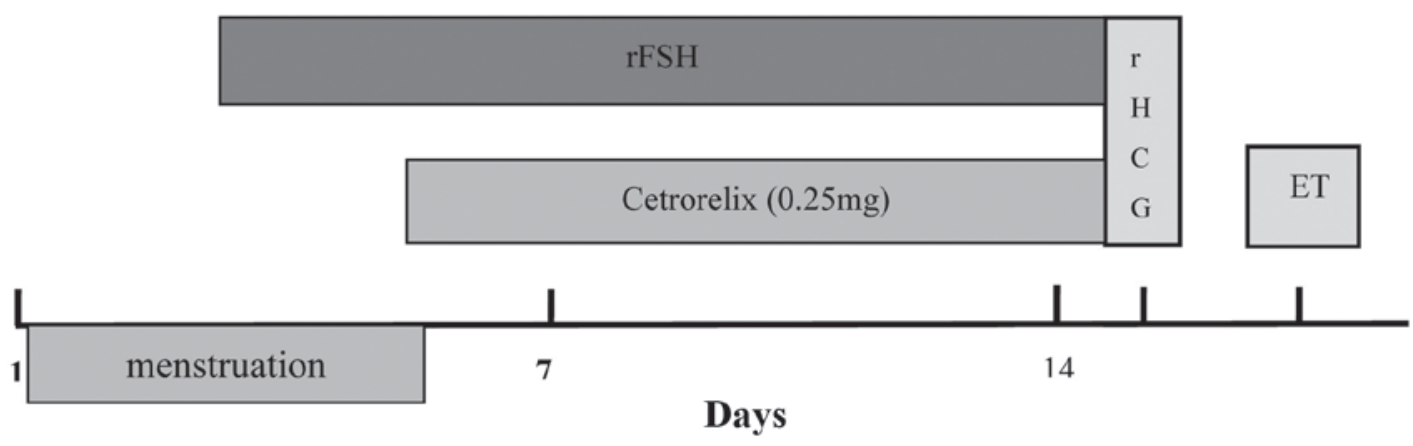

Figure 2. Gonadotropin-releasing hormone antagonist stimulation protocol. rFSH, recombinant follicle-stimulating hormone; rHCG, recombinant human chorionic gonadotropin; ET, embryo transfer.

49 fresh cycles in the standard agonist group (GnRH-a) protocol. The serum gonadotropin (FSH and LH), $\mathrm{E}_{2}$ and $\mathrm{P}$ levels were evaluated beyond the baseline, and the baseline characteristics are depicted in Table I. There were no significant differences in the baseline parameters of age, BMI, FSH, $\mathrm{E}_{2}$ and $\mathrm{P}$ basal hormone profile between the GnRH-A and GnRH-a groups. Furthermore, the mean age was $30.58 \pm 3.74$ years for the GnRH-A group and 29.61 4 4.66 years for the $\mathrm{GnRH}$-a group $(\mathrm{P}=0.220)$, and the mean BMI was $25.24 \pm 3.35 \mathrm{~kg} / \mathrm{m}^{2}$ for the GnRH-A group and $24.21 \pm 3.38 \mathrm{~kg} / \mathrm{m}^{2}$ for the $\mathrm{GnRH}$-a group $(\mathrm{P}=0.133)$. The mean basal hormone profile of FSH was $5.83 \pm 1.35 \mathrm{IU} / 1$ for the GnRH-A group and 5.88 $\pm 1.49 \mathrm{IU} / 1$ for the $\mathrm{GnRH}$-a group $(\mathrm{P}=0.849)$; of $\mathrm{E}_{2}$ was $47.26 \pm 16.92 \mathrm{pg} / \mathrm{ml}$ for the GnRH-A group and 45.27 $\pm 19.7 \mathrm{pg} / \mathrm{ml}$ for the GnRH-a group $(\mathrm{P}=0.591)$; and of $\mathrm{P}$ was $0.47 \pm 0.32 \mathrm{ng} / \mathrm{ml}$ for the GnRH-A group and $0.37 \pm 0.26 \mathrm{ng} / \mathrm{ml}$ for the $\mathrm{GnRH}$-a group $(\mathrm{P}=0.108)$. However, the LH level was significantly different between the two groups: The mean LH was 11.04 $\pm 6.48 \mathrm{IU} / 1$ for the GnRH-A group and 8.15 $\pm 4.98 \mathrm{IU} / 1$ for the GnRH-a group $(\mathrm{P}=0.036)$.

When the two groups were compared, there were no significant differences in the stimulation duration (rFSH days of usage), dose of gonadotrophins or the endometrial thickness on the day of embryo transfer (Table II). However, there were significant differences in the $\mathrm{E}_{2}$ level on the $\mathrm{hCG}$ day $(2,613.2 \pm 945.06$ vs. $4,155.2 \pm 1,367.6 \mathrm{pg} / \mathrm{ml} ; \mathrm{P}<0.001)$, the $\mathrm{P}$ level on the hCG day $(0.68 \pm 0.31$ vs. $0.37 \pm 0.26 \mathrm{ng} / \mathrm{ml} ; \mathrm{P}<0.001)$, the mean number of total oocytes retrieved $(10.50 \pm 2.78$ vs. $13.61 \pm 4.67 ; \mathrm{P}=0.001)$, the mean number of $2 \mathrm{PN}$ oocytes $(5.64 \pm 2.33$ vs. $8.37 \pm 3.70 ; \mathrm{P}<0.001)$, the mean number of oocytes cleaved $(6.38 \pm 2.39$ vs. $11.31 \pm 4.26 ; \mathrm{P}<0.001)$, the mean number of embryos available (3.68 \pm 1.74 vs. $4.92 \pm 2.63$; $\mathrm{P}=0.022)$ and the mean number of embryos transferred (1.90 \pm 0.30 vs. $2.02 \pm 0.14 ; \mathrm{P}=0.014)$.

There was no significant difference in the outcomes of the pregnancy in the two groups, such as the biochemical pregnancy rate [29 (58\%) vs. $31(63.27 \%) ; \mathrm{P}=0.592]$, clinical pregnancy rate [23 (46\%) vs. 25 (51.02\%); $\mathrm{P}=0.617$ ], ectopic pregnancy rate $[2(4 \%)$ vs. $2(4.08 \%) ; \mathrm{P}=0.984]$ and OHSS rate $[4(8 \%)$ vs. $4(8.16 \%) ; \mathrm{P}=0.976]$. The group of patients who received GnRH-A had no more live births than the group of patients who received GnRH-a [17 (34\%) vs. 21 (42.86\%); $\mathrm{P}=0.365]$, either.

Estimated ROC curve for the performance of endometrial thickness and estradiol level on the $h C G$ day in the prediction of a clinical pregnancy. The endometrial thickness on the hCG day ranged between 7 and $14 \mathrm{~mm}$ in the GnRH-A 
Table I. Baseline characteristics (basal hormone profile) of patients in the GnRH-A and GnRH-a groups.

\begin{tabular}{lccc}
\hline & $\begin{array}{c}\text { GnRH-A } \\
\text { group } \\
\text { (50 cycles) }\end{array}$ & $\begin{array}{c}\text { GnRH-a } \\
\text { group } \\
\text { (49 cycles) }\end{array}$ & P-values \\
\hline Age (years) & $30.58 \pm 3.74$ & $29.61 \pm 4.66$ & $0.220^{\mathrm{a}}$ \\
$\mathrm{BMI}\left(\mathrm{kg} / \mathrm{m}^{2}\right)$ & $25.24 \pm 3.35$ & $24.21 \pm 3.38$ & $0.133^{\mathrm{b}}$ \\
$\mathrm{FSH}(\mathrm{IU} / \mathrm{l})$ & $5.83 \pm 1.35$ & $5.88 \pm 1.49$ & $0.849^{\mathrm{b}}$ \\
$\mathrm{LH}(\mathrm{IU} / \mathrm{l})$ & $11.04 \pm 6.48$ & $8.15 \pm 4.98$ & $0.036^{\mathrm{a}}$ \\
$\mathrm{E}_{2}(\mathrm{pg} / \mathrm{ml})$ & $47.26 \pm 16.92$ & $45.27 \pm 19.73$ & $0.591^{\mathrm{b}}$ \\
$\mathrm{P}(\mathrm{ng} / \mathrm{ml})$ & $0.47 \pm 0.32$ & $0.37 \pm 0.26$ & $0.108^{\mathrm{a}}$ \\
\hline
\end{tabular}

a Statistical analysis was performed with use of two independent-sample tests by the Wilcoxon-Mann-Whitney test. ${ }^{\text {bStatistical }}$ analysis was performed with use of Student's t-test. $\mathrm{P}<0.05$ was considered to indicate a statistically significant difference. GnRH-A, gonadotropin-releasing hormone antagonist; GnRH-a, gonadotropin-releasing hormone agonist; BMI, body mass index; FSH, follicle-stimulating hormone; $\mathrm{LH}$, luteinizing hormone; $\mathrm{E}_{2}$, estradiol; $\mathrm{P}$, progesterone.

group and 7 and $16 \mathrm{~mm}$ in the GnRH-a group, which was not a significant difference $(10.18 \pm 1.91$ vs. $10.63 \pm 2.53 ; \mathrm{P}=0.610)$. The plot of the sensitivity-specificity combinations in an ROC space is shown in Figs. 3 and 4. For endometrial thickness, the sensitivity varied between 2.1 and $97.9 \%$ and the specificity between 9.8 and $98 \%$. For the $\mathrm{E}_{2}$ level on the hCG day, the sensitivity varied between 2.1 and $97.9 \%$, and the specificity varied between 2 and $98 \%$.

Analysis of the influence of endometrial thickness on clinical pregnancy, using the ROC (AUC) showed that the AUC was 0.53 [95\% confidence interval (CI): 0.415-0.644], and when the cutoff was $9.75 \mathrm{~cm}$, the sensitivity was $62.5 \%$ and the specificity was $43.1 \%$. Additionally, the Youden index was 0.056 (Fig. 3). The results of the influence of the $E_{2}$ level on the hCG day $(\mathrm{pg} / \mathrm{ml})$ on the clinical pregnancy rate, using the ROC (AUC), showed that the AUC was 0.573 (95\% CI: 0.459-0.687). In addition, when the cutoff was $2,984.5 \mathrm{pg} / \mathrm{ml}$, the sensitivity was $68.8 \%$ and the specificity $52.9 \%$. Additionally, the Youden index was 0.217 (Fig. 4). The results of the ROC curve analysis demonstrated that neither the endometrial thickness nor the $\mathrm{E}_{2}$ level $(\mathrm{pg} / \mathrm{ml})$ on the hCG day had the best sensitivity and specificity for predicting clinical pregnancy.

\section{Discussion}

It is well known that $\mathrm{COH}$ is an important component of IVF-ET technology and that different $\mathrm{COH}$ protocols can result in different ovarian responses, even in the same patient. The GnRH agonist long protocol has been used in the majority of IVF treatment cycles since the 1980s. Currently, a number of studies have shown that the clinical pregnancy rate and the live birth rate are not significantly different in PCOS patients receiving agonist treatment compared with those receiving antagonist treatment (8-10). However, Orvieto et al (11) clearly observed a significantly higher clinical pregnancy rate with a $\mathrm{GnRH}$ agonist suppressive protocol than in a GnRH antagonist protocol, which is in accordance with certain meta-analysis literature (12).

It has been reported that in poor responders, the number of mature oocytes, the cycle cancellation rate and clinical pregnancy rate per cycle initiated were not significantly different when the GnRH agonist regimen was compared to the $\mathrm{GnRH}$ antagonist protocol. However, the GnRH antagonist protocol was associated with a significantly higher number of retrieved oocytes (13). In fact, with multifollicular development, the occurrence of cyst formation at the initiation of stimulation and the increased risk of OHSS following the administration of hCG represent the main difficulties of ovulation induction in women with PCOS. Additionally, an excessive response to gonadotropin stimulation is associated with painful oocyte retrieval, abdominal discomfort and even an increased chance of cycle cancellation. Since severe OHSS is a life-threatening complication of ovulation induction, it should be considered to be important when deciding the treatment protocol for women with PCOS. It is reported that the GnRH antagonist protocol is safer than the agonist protocol because it could enable the use of GnRH agonist instead of hCG, to trigger ovulation, which is associated with a significant reduction in the occurrence of severe OHSS. Furthermore, use of a GnRH antagonist represents a valid alternative to use of a $\mathrm{GnRH}$ agonist to prevent a premature LH surge in women with PCOS (14). In the present study, the data showed that the OHSS rate was not significantly different between the two groups, which possibly stemmed largely from fresh embryo transplant cycle cancellation (in which all of the embryos that were available would be cryopreserved) with the potential of OHSS, particularly in the GnRH-a protocol.

It is reported that the extremely high levels of $\mathrm{E}_{2}$ that are observed impair both the embryo quality and endometrial receptivity, which threatens embryo implantation (15). The level of $\mathrm{E}_{2}$ in women with PCOS is significantly higher in the GnRH agonist than that in the GnRH antagonist protocol (16). Manno et al (17) reported that in cycles in which $\mathrm{E}_{2}$ levels are $\leq 3,000 \mathrm{pg} / \mathrm{ml}$, there was only a trend that favors the implantation rate in the $\mathrm{GnRH}$ agonist, while in cycles with $\mathrm{E}_{2}$ levels $\geq 3,000 \mathrm{pg} / \mathrm{ml}$ the implantation rate was significantly higher in the GnRH antagonist protocol. The results of a systematic review (18) revealed that IVF-ET patients with supposed normal responses instead of PCOS have significantly lower $\mathrm{E}_{2}$ values on the day of hCG, number of oocytes retrieved and incidence of OHSS with the GnRH antagonist protocol compared with those of the GnRH agonist protocol. Furthermore, the endometrial thickness on the day of hCG and the live birth rate were similar in the above two groups. However, whether there was a difference in the clinical pregnancy rate between the two groups remained uncertain.

In the present study, there were significantly higher $\mathrm{E}_{2}$ levels on the hCG day in the GnRH-a group compared with the GnRH-A group. Additionally, the GnRH-a protocol appeared to be more effective compared with the GnRH-A protocol in terms of the number of total oocytes retrieved, number of 2 PN oocytes, number of oocytes cleaved, number of embryos transferred and number of embryos available. However, it appears that there was no significant difference in either the clinical pregnancy rate or the live births per woman, although the latter was higher in the GnRH-a group. 
Table II. Comparison of the two groups regarding stimulation characteristics and outcome in IVF.

\begin{tabular}{lccr}
\hline Variables & GnRH-A group & GnRH-a group & P-values \\
\hline Stimulation duration (days) & $9.54 \pm 1.40$ & $9.65 \pm 1.85$ & $0.744^{\mathrm{a}}$ \\
Dose of gonadotrophins (IU) & $1,878.50 \pm 400.52$ & $1,768.4 \pm 501.13$ & $0.119^{\mathrm{a}}$ \\
$\mathrm{E}_{2}$ on hCG day (pg/ml) & $2,613.2 \pm 945.06$ & $4,155.2 \pm 1367.6$ & $<.001^{\mathrm{b}}$ \\
P on hCG day (ng/ml) & $0.68 \pm 0.31$ & $0.37 \pm 0.26$ & $<.001^{\mathrm{a}}$ \\
EM on hCG day (cm) & $10.18 \pm 1.91$ & $10.63 \pm 2.53$ & $0.610^{\mathrm{a}}$ \\
No. of oocytes retrieved & $10.50 \pm 2.78$ & $13.61 \pm 4.67$ & $0.001^{\mathrm{a}}$ \\
No. of 2 PN oocytes & $5.64 \pm 2.33$ & $8.37 \pm 3.70$ & $<0.001^{\mathrm{b}}$ \\
No. of oocytes cleaved & $6.38 \pm 2.39$ & $11.31 \pm 4.26$ & $<0.001^{\mathrm{a}}$ \\
No. of embryos available & $3.68 \pm 1.74$ & $4.92 \pm 2.63$ & $0.022^{\mathrm{a}}$ \\
No. of embryos transferred & $1.90 \pm 0.30$ & $2.02 \pm 0.14$ & $0.014^{\mathrm{a}}$ \\
Biochemical pregnancy rate, $\mathrm{n}(\%)$ & $29(58)$ & $31(63.27)$ & 0.592 \\
Clinical pregnancy rate, $\mathrm{n}(\%)$ & $23(46)$ & $25(51.02)$ & 0.617 \\
Ectopic pregnancy rate, $\mathrm{n}(\%)$ & $2(4)$ & $2(4.08)$ & 0.984 \\
Live birth rate, $\mathrm{n}(\%)$ & $17(34)$ & $21(42.86)$ & 0.365 \\
OHSS rate, $\mathrm{n}(\%)$ & $4(8)$ & $4(8.16)$ & 0.976 \\
\hline
\end{tabular}

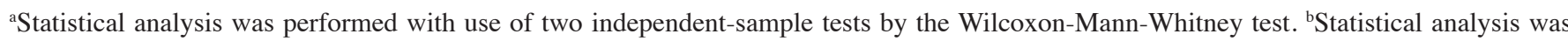
performed with use of Student's t-test. $\mathrm{P}<0.05$ was considered to indicate a statistically significant difference. IVF, in vitro fertilization; GnRH-A, gonadotropin-releasing hormone antagonist; GnRH-a, gonadotropin-releasing hormone agonist; $\mathrm{E}_{2}$, estradiol; hCG day, day that human chorionic gonadotropin was administered; P, progesterone; EM, endometrial thickness on day of embryo transfer; PN, pronuclei; OHSS, ovarian hyperstimulation syndrome. Live birth was defined by the delivery of a live-born infant.

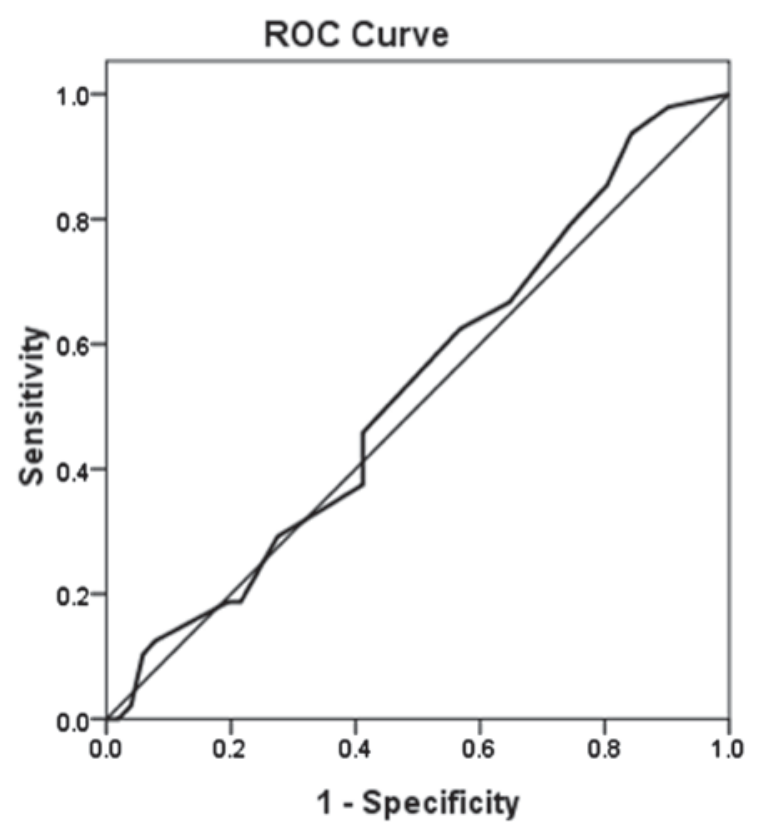

Figure 3. Estimated ROC curve and sensitivity-specificity points for the performance of endometrial thickness on the hCG day in the prediction of a clinical pregnancy. ROC, receiver operating characteristic; $\mathrm{hCG}$ day, day that human chorionic gonadotropin was administered.

A successful pregnancy requires implantation, which requires healthy endometrial receptivity, which is one of the most important factors in predicting pregnancy following IVF-ET. The endometrial thickness is measured in the midsagittal plane via transvaginal ultrasound, and it has been utilized as an individual indicator for endometrial receptivity $(19,20)$.

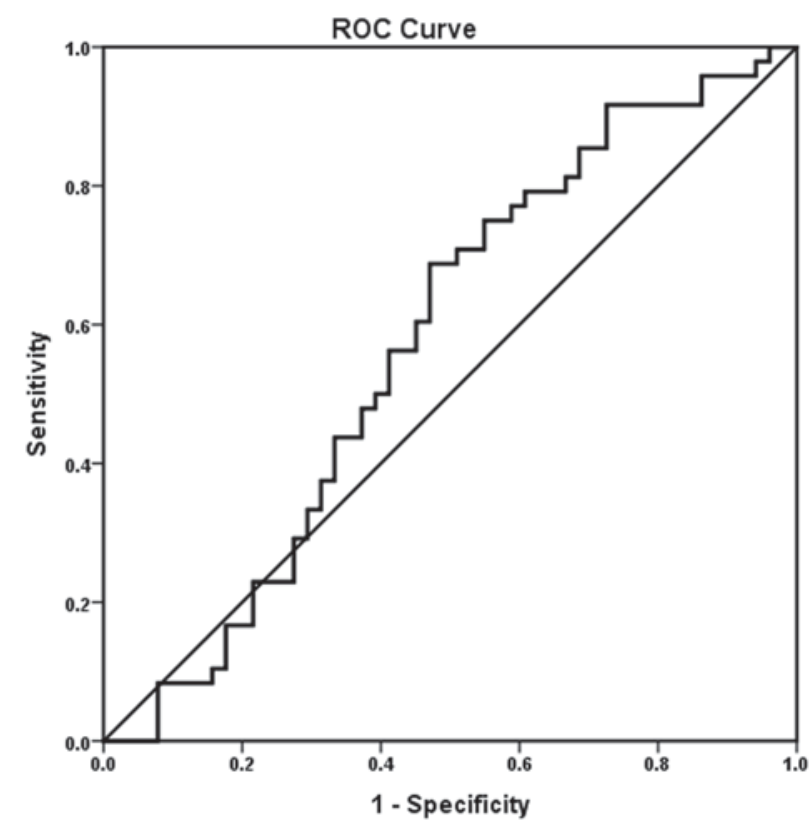

Figure 4. Estimated ROC curve and sensitivity-specificity points for the performance of estradiol level on the hCG day in the prediction of a clinical pregnancy. ROC, receiver operating characteristic; hCG day, day that human chorionic gonadotropin was administered.

It has been revealed that the clinical pregnancy rate is $<30 \%$, and the live birth rate is $<20 \%$ in patients with an endometrium thickness of $\leq 7 \mathrm{~mm}$ (21). Additionally, no significant difference has been identified in the clinical pregnancy and implantation rates with endometrial thicknesses in the 8-14 mm range compared with $>14 \mathrm{~mm}$ (22). 
Currently, no conclusive cut-off value has been established for endometrial thickness. In the present study, it was demonstrated that the endometrial thickness on the hCG day ranged between 7 and $16 \mathrm{~mm}$ in both the GnRH-A and GnRH-a groups, which exhibited no significant difference between groups, or in either the clinical pregnancy or live birth rates per patient. Furthermore, the ROC curve analysis demonstrated that neither the endometrial thickness nor $\mathrm{E}_{2}$ level on the hCG day had the best specificity and sensitivity for predicting a clinical pregnancy. However, additional large studies should be performed to elucidate the role of the endometrial thickness and $\mathrm{E}_{2}$ level on the hCG day and in women with PCOS undergoing GnRH antagonist or GnRH agonist treatment.

In conclusion, in this series of women with PCOS who were undergoing IVF-ET cycles, GnRH antagonist decreased oocyte retrieval, the number of oocytes cleaved, the number of embryos available and the number of embryos transferred. However, a GnRH antagonist protocol was associated with neither a significantly lower clinical pregnancy rate nor live birth rate compared with that of the GnRH agonist suppressive protocol. With regard to safety, the advantage of the GnRH antagonist protocol is the possibility of administering a GnRH agonist instead of hCG for ovulation triggering with the consequent elimination of severe OHSS, although a number of controversial issues remain concerning the use of $\mathrm{GnRH}$ antagonist in IVF. Above all, the nRH antagonist approach has rendered IVF-ET a patient-friendly and safer procedure with high efficacy compared with that of the GnRH agonist approach. Additionally, future studies should show that GnRH antagonists ensure good reproductive and clinical outcomes with very little risk of OHSS development.

\section{Acknowledgements}

The study was supported by the Shangdong Provincial Natural Science Foundation of China (grant nos. ZR2010CQ035 and 2014ZRA13020) and the Shandong Provincial Medical and Health Science and Technology Development Project, China (grant no. 2015WS0377). The authors thank the administrative staff in the Department of Reproductive Medicine in Linyi People's Hospital for support.

\section{References}

1. Rotterdam ESHRE/ASRM-Sponsored PCOS Consensus Workshop Group: Revised 2003 consensus on diagnostic criteria and long-term health risks related to polycystic ovary syndrome. Fertil Steril 81: 19-25, 2004.

2. Griesinger G, Diedrich K, Tarlatzis BC and Kolibianakis EM: GnRH-antagonists in ovarian stimulation for IVF in patients with poor response to gonadotrophins, polycystic ovary syndrome and risk of ovarian hyperstimulation: A meta-analysis. Reprod Biomed Online 13: 628-638, 2006.

3. Dell'Aquila ME, Ambruosi B, De Santis T and Cho YS: Mitochondrial distribtuion and activity in human mature oocytes: Gonadotropin-releasing hormone agonist versus antagonist for pituitary down-regulation. Fertil Steril 91: 249-255, 2009.
4. Fahy UM, Cahill DJ, Wardle PG and Hull MG: In vitro fertilization in completely natural cycles. Hum Reprod 10: 572-575, 1995.

5. Huirne JA and Lambalk CB: Gonadotrophin-releasing hormone receptor antagonists. Lancet 358: 1793-1803, 2001.

6. Orvieto R: Can we eliminate severe ovarian hyperstimulation syndrome? Hum Reprod 20: 320-322, 2005.

7. Cooper TG, Noonan E, von Eckardstein S, Auger J, Baker HW, Behre HM, Haugen TB, Kruger T, Wang C, Mbizvo MT and Vogelsong KM: World health organization reference values for human semen characteristics. Hum Reprod Update 16: 231-245, 2010.

8. Kolibianakis EM, Collins J, Tarlatzis BC, Devroey P, Diedrich K and Griesinger G: Among patients treated for IVF with gonadotrophins and GnRH analogues, is the probability of live birth dependent on the type of analogue used? A systematic review and meta-analysis. Hum Reprod Update 12: 651-671, 2006.

9. Al-Inany HG, Youssef MA, Aboulghar M, Broekmans F, Sterrenburg M, Smit J and Abou-Setta AM: Gonadotrophinreleasing hormone antagonists for assisted reproductive technology. Cochrane Database Syst Rev 11: CD001750, 2011.

10. Xiao J, Chen S, Zhang $\mathrm{C}$ and Chang S: Effectiveness of GnRH antagonist in the treatment of patients with polycystic ovary syndrome undergoing IVF: A systematic review and meta analysis. Gynecol Endocrinol 29: 187-191, 2013.

11. Orvieto R, Meltcer S, Homburg R, Nahum R, Rabinson J and Ashkenazi J: What is the preferred GnRH analogue for polycystic ovary syndrome patients undergoing controlled ovarian hyperstimulation for in vitro fertilization? Fertil Steril 91 (Suppl 4): S1466-S1468, 2009.

12. Al-Inany HG, Abou-Setta AM and Aboulghar M: Gonadotrophin releasing hormone antagonists for assisted conception: A Cochrane review. Reprod Biomed Online 14: 640-649, 2007.

13. Sbracia M, Colabianchi J, Giallonardo A, Giannini P, Piscitelli C, Morgia F, Montigiani M and Schimberni M: Cetrorelix protocol versus gonadotropin-releasing hormone analog suppression long protocol for superovulation in intracytoplasmic sperm injection patients older than 40. Fertil Steril 91: 1842-1847, 2009.

14. Al-Inany HG, Youssef MA, Aboulghar M, Broekmans F, Sterrenburg M, Smit J and Abou-Setta AM: GnRH antagonists are safer than agonists: An update of a Cochrane review. Hum Reprod Update 17: 435, 2011.

15. de Ziegler D, Fanchin R, de Moustier B and Bulletti C: The hormonal control of endometrial receptivity: Estrogen (E2) and progesterone. J Reprod Immunol 39: 149-166, 1998.

16. Kdous M, Chaker A, Bouyahia M, Zhioua F and Zhioua A: Increased risk of early pregnancy loss and lower live birth rate with GNRH antagonist vs. long GNRH agonist protocol in PCOS women undergoing controlled ovarian hyperstimulation. Tunis Med 87: 834-842, 2009 (In French).

17. Manno M, Cervi M,Zadro D, Fuggetta G, Adamo V and Tomei F: Different ART outcomes at increasing peak estradiol levels with long and antagonist protocols: Retrospective insights from ten years experience. J Assist Reprod Genet 28: 693-698, 2011.

18. Xiao JS, Su CM and Zeng XT: Comparisons of GnRH antagonist versus $\mathrm{GnRH}$ agonist protocol in supposed normal ovarian responders undergoing IVF: A systematic review and meta-analysis. PLoS One 9: e106854, 2014.

19. Friedler S, Schenker JG, Herman A and Lewin A: The role of ultrasonography in the evaluation of endometrial receptivity following assisted reproductive treatments: A critical review. Hum Reprod Update 2: 323-325, 1996.

20. Dekel N, Gnainsky Y, Granot I and Mor G: Inflammation and implantation. Am J Reprod Immunol 63: 17-21, 2010.

21. Wu Y, Gao X, Lu X, Xi J, Jiang S, Sun Y and Xi X: Endometrial thickness affects the outcome of in vitro fertilization and embryo transfer in normal responders after GnRH antagonist administration. Reprod Biol Endocrinol 12: 96, 2014.

22. Yoeli R, Ashkenazi J, Orvieto R, Shelef M, Kaplan B and Bar-Hava I: Significance of increased endometrial thickness in assisted reproduction technology treatments. J Assist Reprod Genet 21: 285-289, 2004. 